\title{
Image Mosaic Algorithm Based on Relative Orientation
}

\author{
Zhang Yi-fei ${ }^{*}$ and $\mathrm{He} \mathrm{Yi}$
}

\author{
Henan University of Urban Construction, Pingdingshan, Henan, 467036, China
}

\begin{abstract}
The paper proposes a new image mosaic algorithm based on relative orientation. In view of image registration errors in existing image mosaic algorithms, the relative orientation method is introduced. The parameters of relative orientation are obtained by parsing stereo image pairs, and the transformation model of ideal image pairs are generated. The paper uses mean parallax method to register the ideal image pairs in one-dimensional way, and finally realizes the image mosaic algorithm based on ideal image pairs model. Experiment results show that the method can effectively reduce the image registration errors and the calculation of image registration, and also reduce the difficulty of image mosaic of threedimensional objects on two-dimensional plane. Therefore, this method is an efficient image mosaic algorithm.
\end{abstract}

Keywords: Ideal image pair, image mosaic, parall mean, registration error, relative orientation.

\section{INTRODUCTION}

The technology of image mosaic is an important branch of the technology of digital image processing. Through the process of registration and pixel fusion of pictures with overlapping regions in the same scene, a new image with wider view of the same resolution can be made [1]. This technology can be applied not only to obtaining images with wider view, but also to video surveillance, virtual reality, remote sensing and medical science, etc. It is generally acknowledged that image mosaic includes five steps, which are image preprocessing, building conversion model, unifying coordinate transformation, and image registration and fusion. Among them, image registration is the core technology of image mosaic, and the former directly determines the result of the latter [2]. Differences of shooting angle, light intensity and resolution ratio may bring to differences of translation, spinning and perspective shape change of images to be jointed. The purpose of image registration is to find a transformational model which can best describe the mapping relation between images to be jointed, and to corresponded homologous image points in two images to each other. Algorithms for image registration are various, which can be classified into three types: image registration algorithms based on gray information [3], image registration algorithms based on feature [4] and image registration algorithms based on transform domain [5] As for the two-dimensional image mosaic algorithm, it has advantages as well as the defect of considerable registration error.

This paper particularly studies and analyzes image mosaic based on the principle of photographic imaging, and finds out that the primary causes for registration error are the vertical parallax(difference in vertical coordinates of homologous image points) and the horizontal parallax(difference in horizontal coordinates of homologous image points) generate in the process of shooting images. And based on the above findings, relative orientation model in photogrammetry is introduced to image mosaic. The new algorithm designs the ideal image pair of transformation model algorithmically, and proposes a new method of image registration by making use of the horizontal parallax mean. The working process is as follows: firstly, relative orientation technology is adopted to generate ideal image pairs; then, match these ideal image pairs to homologous image points on the basis of one dimension so as to eliminate the horizontal parallax of the image pairs to be jointed $[6,7]$. Experiment results show that the new method can effectively reduce the image registration errors and the stitching result is obviously better that other image mosaic algorithm.

\section{ALGORITHM BASIS}

\subsection{Feature Matching}

The purpose of feature matching is to find the points or regions with similar features of the images to be jointed and get the matching points in accordance with matching method, and then the transformation relation of two images is available. In this paper, the feature matching is conducted by combining bilateral matching with the nearest neighbor algorithm $[8,9]$.

The matching process is as follows:

(1) Select the feature point PL of Image A, and traverse all the feature points of image $\mathrm{B}$, then find point $\mathrm{PR}$ which is the nearest one to PL and PR1 which is next to the nearest one.

(2) If the value of PR/PR1is less than the set threshold value, then PR is the candidate matching point for PL, and move to step (3); otherwise, it proves that there is no matching point for $\mathrm{PL}$ in image $\mathrm{B}$. 


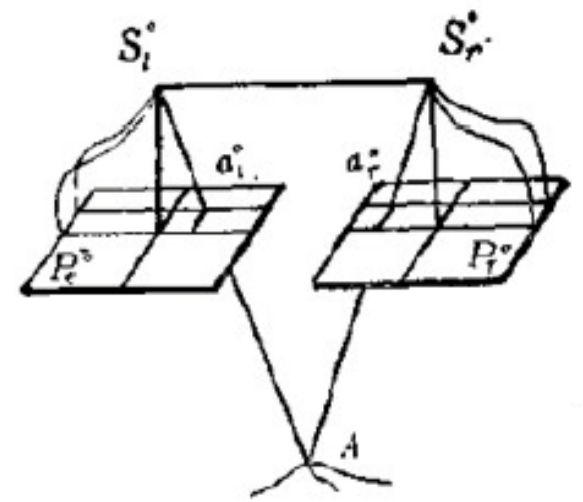

Fig. (1). Sketch of Ideal Image Pair.

(3) Set PR as the corresponding point, and traverse all the feature points in image A, then find PR' which is the nearest one to PR and PR1'which is next to the nearest one. If the value of PR'PR1'is less than the set threshold value, then move to step (4).

(4) Decide if PL and PL' is the same point, if so, record PL and PR are matching point pair; if not, then there is no corresponding matching point for PL in image B; Afterwards, traverse all the feature points in image $\mathrm{A}$ and end the matching algorithm.

\subsection{Relative Orientation}

Relative orientation is the basis of building the ideal image pairs for transformation model. By constantly reconstituting the shooting rays of two images, corresponding image rays will cross each other and proportional geometric model of objects will be generated $[10,11]$. As for separate relative orientation, photographic base is regarded as the criterion and the $X$ axis of auxiliary space coordinate system. Relative orientation is realized by the angle element movement of two images, that is make the center of projection of two images spindle and regain the relation of the shooting rays of two images. There are six angle parameters of separate relative orientation: $\omega_{1}, \phi_{1}, \kappa_{1}, \omega_{2}, \phi_{2}, \kappa_{2}$. These angle parameters are angles at which two images revolve to make corresponding image rays cross. Since photographic base is the $X$ axis of auxiliary space coordinate system, then $\omega_{1}=0$. In order to solve the parameters, the ligature between the shooting points $S_{1}, S_{2}$ and the image points of object A on two images $a_{1}\left(x_{1}, y_{1}\right), a_{2}\left(x_{2}, y_{2}\right)$ (image points of object $\mathrm{A}$ on two images)must be on the same plane. If auxiliary space coordinates of points $a_{1}, a_{2}$ are $\left(X_{1}, Y_{1}, Z_{1}\right),\left(X_{2}, Y_{2}, Z_{2}\right)$, then the coplanar condition is:

$$
F=\left|\begin{array}{ccc}
B_{X} & 0 & 0 \\
X_{1} & Y_{1} & Z_{1} \\
X_{2} & Y_{2} & Z_{2}
\end{array}\right|=0
$$

Use Taylor formula to linearly unfold formula (1), and deduce the resolving equation of relative orientation algorithm.

$$
\begin{aligned}
& q=-\frac{X_{2} Y_{2}}{Z_{2}} N_{2} d \phi-\left(Z_{2}+\frac{Y_{2}^{2}}{Z_{2}}\right) N_{2} d \omega+ \\
& X_{2} N_{2} d \kappa+\mathrm{B}_{X} d \mu-\frac{Y_{2}}{Z_{2}} B_{X} d v
\end{aligned}
$$

There are five parameters to be resolved. When six corresponding matching points are gained, then least square method is used to get iterative solution and the unknown parameters of relative orientation.

\subsection{Ideal Image Pair}

Ideal image pair (also named epipolar ray image) is stereo pair which is horizontal and with horizontal photographic base (ligature between two shooting sites). It is an image pair with horizontal parallax but not vertical parallax $[7,12]$. In ideal image pair, whether there is altitude difference between objects or not, the vertical coordinates of all corresponding image points are always equal, i.e., vertical parallax is zero (as is shown in Fig. 1).

Suppose, the original image is $P$, the ideal image pair is $P^{\prime}$, the coordinate of image point $A$ in image $P$ is $(x, y)$, the coordinate of image point $A$ in ideal image pair $P^{\prime}$ is $(u, v)$, then the corresponding relation of coordinates $(x, y)$ and $(u, v)$ can be expressed as:

$$
\left\{\begin{array}{l}
x=-f \frac{a_{1} u+b_{1} v-f c_{1}}{a_{3} u+b_{3} v-f c_{3}} \\
y=-f \frac{a_{2} u+b_{2} v-f c_{2}}{a_{3} u+b_{3} v-f c_{3}}
\end{array}\right.
$$

Since the horizontal parallax of ideal image pair is always zero, introducing ideal image pair as transformation model to image mosaic can effectively remove the vertical parallax of images to be jointed, simplify image registration and reduce registration errors as well.

\section{IMAGE MOSAIC ALGORITHM BASED ON REL- ATIVE ORIENTATION}

The image mosaic algorithm proposed in this paper makes use of the principle of photographic imaging and brings separate relative orientation in image mosaic. This algorithm rebuilds the constrained relation between shooting rays of image pairs and turns the images to be jointed into ideal image pairs, and then image registration is conducted by using horizontal parallax mean.

\subsection{Generating Ideal Image Pair}

The first step is to detect all the feature points with SURF algorithm [13] and get the feature points of the images to be 
jointed. Then, the improved bilateral matching algorithm is used to match all the feature points and initial matching point pairs are available. At last, RANSAC algorithm [14] is adopted to remove mismatching points and finally get the correct matching point pairs. Pick up six pairs of corresponding image points, use formula (2) to get iterative solution on the basis of least square method, and the parameters $\phi_{1}, \kappa_{1}, \omega_{2}, \phi_{2}, \kappa_{2}$ of relative orientation can be solved.

The rotation matrix $\mathrm{R}$ of transformation model is obtained based on the solved parameters of relative orientation.

$$
R=R_{\phi} R_{\omega} R_{\kappa}=\left[\begin{array}{lll}
a_{1} & a_{2} & a_{3} \\
b_{1} & b_{2} & b_{3} \\
c_{1} & c_{2} & c_{3}
\end{array}\right]
$$

As the characters of separate orientation show, after the relative orientation of separate image pair, the plane of delineation in two images is parallel to the photographic base, thus two images turn to horizontal image pair. After the rotation matrix composed of relative orientation parameters is gained, the original images will be resampled to get the coordinate $(x, y)$ of point $\mathrm{P}$ in original image. By making use of formula (3), the corresponding of coordinate $(u, v)$ of ideal image pair can also be obtained, consequently new ideal image pair which is parallel to the photographic base is generated.

\subsection{Registration of Ideal Image Pair with Horizontal Parallax Mean}

After the ideal image pair is generated, one-dimensional registration should be conducted to corresponding image points. In practical application, horizontal parallax caused by altitude difference displacement is not evident. The influence of altitude difference displacement on horizontal parallax weakens especially when ideal image pair is generated. For this reason, horizontal parallax mean serves as the overall horizontal parallax value to conduct image registration. The steps are as follows.

(1) Calculate horizontal parallax mean Dis, resolve the coordinate of matching points on ideal image pair with separate relative orientation algorithm. After remove the maximum and minimum value of horizontal parallax, horizontal parallax mean of the rest matching point pairs is calculated.

(2) The plane on which the horizontal parallax mean locates is set as the base level, i.e., the plane on which the matching points with the same the horizontal parallax and the horizontal parallax mean locates. Then, determine the overlapping region of the pictures to be jointed on the basis of horizontal parallax mean.

(3) Displace ideal image pair to accomplish the registration of ideal image pair.

Suppose in the relative ideal image pair, the coordinate of the corresponding image point in the left image is $P(l x, l y)$ and the coordinate in the right image is $P(r x, r y)$. Then, the horizontal parallax is $D i s_{-} t=l x-r x$, and the registration error of this matching point pair is Dis_t-Dis. Count up all the registration error of the matching points and take the average, which is the overall registration error. It is actually the weighted average of horizontal parallax. The formula is as follows.

$d_{\text {total }}=\frac{\sum_{i=1}^{n}\left|D i s_{-} t_{i}-D i s\right|}{n}$

To judge if horizontal parallax mean can be used as the criterion of jointing ideal image pairs depends on whether the maximum of registration error between image pairs is larger than the set threshold value $\mathrm{T}$ (set in accordance with practical situation). For instance, $\max \left(D_{i} \_t_{i}-D i s\right) \leq \tau$.

\subsection{Measurement of Registration Error}

In ideal image pairs, even though vertical coordinates of corresponding image points are always equal and vertical parallax is zero, horizontal parallax still exists. Suppose the image points of object A on the left and right image are respectively $a_{l}, a_{r}$, while the image points of another object $A_{0}$ on the left and right image are respectively $a_{0 l}, a_{0 r}$. Turn $a_{l}, a_{r}, a_{0 l}, a_{0 r}$ into rectangular coordinate system, then the following formula can be obtained.

$$
\left\{\begin{array}{l}
x_{l}-x_{0 l}=x_{l} \frac{h}{H_{T}} \\
x_{r}-x_{0 r}=x_{r} \frac{h}{H_{T}}
\end{array}\right.
$$

In the formula, $h$ stands for elevation and $H_{T}$ stand for the distance between object points and shooting points. When the second formula is subtracted from the first one, then the following new formula can be obtained.

$$
\left(x_{l}-x_{r}\right)-\left(x_{0 l}-x_{0 r}\right)=\left(x_{l}-x_{r}\right) \frac{h}{H_{T}}
$$

The difference between abscissas of corresponding image points is called horizontal parallax $p$.

$$
p=x_{l}-x_{r}
$$

The horizontal parallax between images with altitude difference displacement is named $\Delta p$.

$\Delta p=\left(x_{l}-x_{r}\right)-\left(x_{0 l}-x_{0 r}\right)$

Then formula (6) can be expressed as follows:

$$
\Delta p=p \frac{h}{H_{T}}
$$



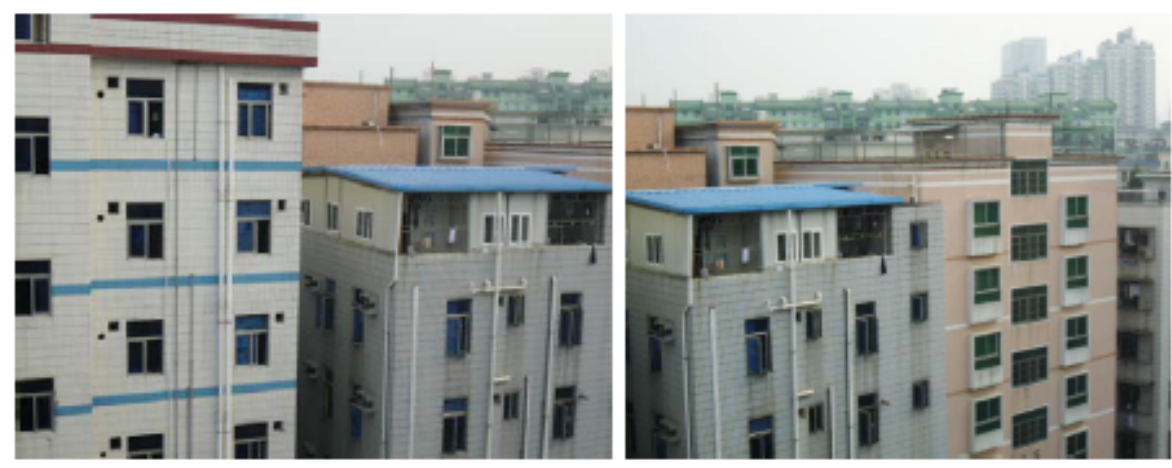

Fig. (2). Images to be Jointed.
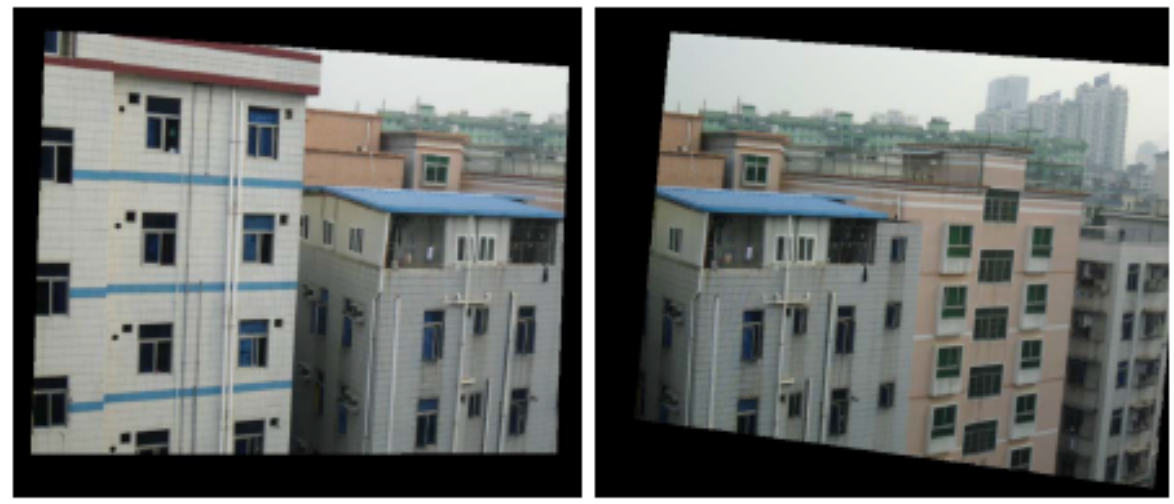

Fig. (3). Ideal Image Pair.

The calculation process shows that when the object is a three-dimensional one, then different points of the object with different elevations have different horizontal parallax in ideal image pair. The size of difference in horizontal parallax is determined by elevations, and this is the embodiment of altitude difference displacement in ideal image pair. When the maximum horizontal parallax of corresponding image points in ideal image pair exceeds a certain limit, the registration error in ideal image pair also exceeds a certain limit. Under this condition, ideal image pair cannot be jointed. For this reason, horizontal parallax can be adopted to measure registration error to quantitatively analyze registration error.

\section{EXPERIMENT AND ANALYSIS}

\subsection{Experiment Process}

The following images (Fig. 2) are chosen to experiment image mosaic and to test the validity of the image mosaic algorithm based on relative orientation.

In the phase of selecting feature points, SURF-64 algorithm is used to detect respectively 640 and 600 feature points from two images. By adopting the improved feature points matching algorithm, 162 pairs of matching point pairs are obtained. Then the mispairing points are got rid of by RANSAC algorithm, and 157 pairs of matching points are finally obtained.
By making use of the 157 pairs of matching point pairs and taking the resolving steps of separate relative orientation parameters, the rotation matrix of two images can be obtained. Afterwards, ideal image pairs are available as well, which is shown in Fig. (3).

The next step is to joint the image pairs by adopting the method of horizontal parallax mean. For lack of space, part of the coordinates and horizontal parallax of matching point pairs is listed in Table $\mathbf{1}$.

According to algorithm of horizontal parallax mean, the horizontal parallax mean of the set of matching point pairs Dis $=323$ pixel, i.e., choose plane that formed by object points numbered 36, 48 and 92 to serve as the base level. In the experiment, the horizontal parallax mean 323pixel serves as the displacement between image pairs to conduct image registration. The registration effect is shown in Fig. (4).

\subsection{Experiment Analysis}

The registration error of matching point pairs is shown in Table 2. From the table, it is clear to see that the overall registration error of horizontal parallax mean of the selected 157 pairs of matching points is between 1 to 2 pixels. In the generated ideal image pairs, the maximum and minimum of horizontal parallax is respectively 318 pixels and 326 pixels. The value range of horizontal parallax mean is between 322 to 324 pixels, thus the maximum registration error between 


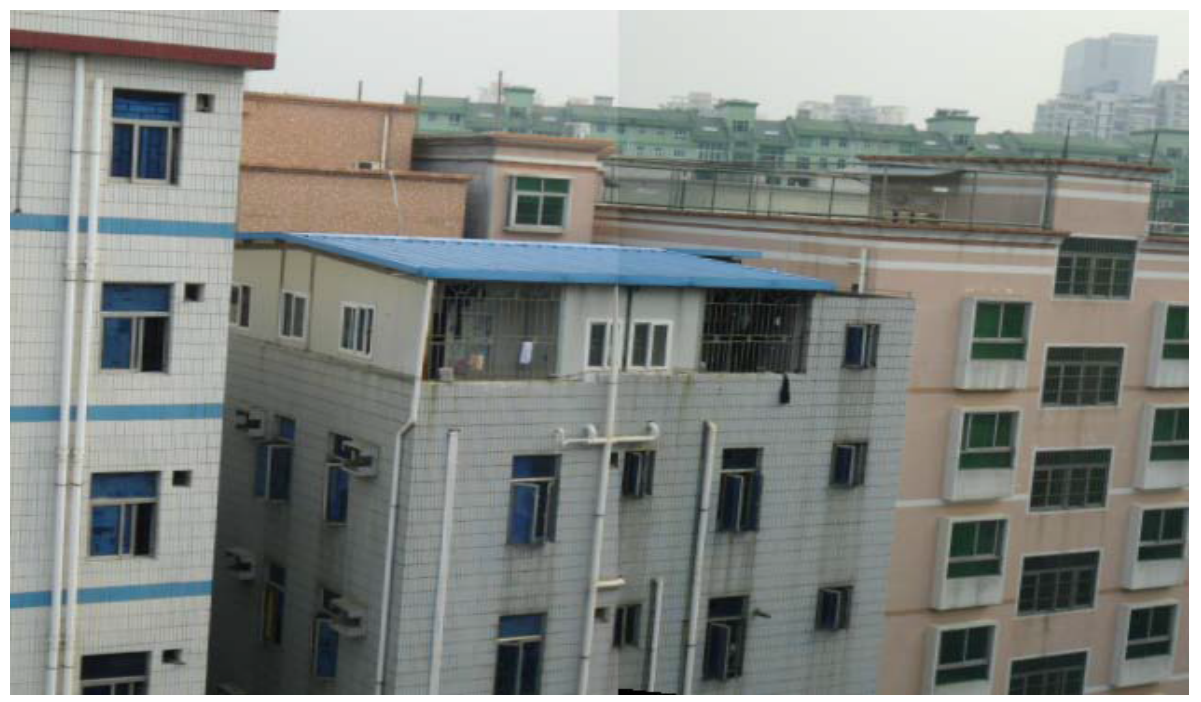

Fig. (4). Registration Effect.

Table 1. Part of the coordinates of matching point pairs.

\begin{tabular}{|c|c|c|c|}
\hline No & Left Coordinates & Right Coordinates & Horizontal Parallax \\
\hline \hline 0 & $(451,418)$ & $(130,418)$ & 321 \\
\hline 12 & $(473,409)$ & $(154,409)$ & 319 \\
\hline 36 & $(476,315)$ & $(193,315)$ & 324 \\
\hline 40 & $(518,280)$ & $(99,252)$ & 323 \\
\hline 48 & $(422,252)$ & $(173,87)$ & 325 \\
\hline 88 & $(498,87)$ & $(252,59)$ & 323 \\
\hline 92 & $(575,59)$ & $(185,47)$ & 324 \\
\hline 96 & $(509,47)$ & $(104,398)$ & 320 \\
\hline 124 & $(424,398)$ & & \\
\hline
\end{tabular}

Table 2. Registration error of matching point pair.

\begin{tabular}{|c|c|}
\hline Matching Point Pair & Parallax Mean/Parallax Minimum/Parallax Maximum/Most Parallax/Registration Error \\
\hline \hline 157 & $323 / 318(2) / 326(3) / 324(45) / 1.38217$ \\
\hline
\end{tabular}

ideal image pairs is 6 pixels. This result is in accordance with the error range of adopting horizontal parallax to conduct image mosaic.

To highlight the seam crossing, each half of the two images in the overlapping region are displayed in image 4 . Because of the obvious luminance difference in two images, it is intuitive to distinguish the margin of the seam crossing from each other. Scan the margin of the seam crossing, no malposition or distortion can be found. This also proves that the new algorithm not only improves the jointing effect but also reduces the calculation amount.
Registration error generated in algorithm based on separate relative orientation is mainly from horizontal parallax difference caused by altitude difference displacement of objects. For this reason, all the corresponding image points in overlapping region cannot be conducted registration. In order to analyze the registration error of the new algorithm, the data of 8 corresponding image points with separate relative orientation are collected; then, calculate the maximum horizontal parallax difference under the circumstance of horizontal base line, i.e., component $B y=0, B z=0$; component $B x$ and angle $w$ take different values. In the experiment, the fixed 
Table 3. Registration error of corresponding image points with different parameters.

\begin{tabular}{|c|c|c|c|c|c|c|c|}
\hline \multirow{2}{*}{ Anglew } & \multirow{2}{*}{$B x / H$} & \multicolumn{6}{|c|}{$h / H$} \\
\hline & & 0.5 & 0.4 & 0.3 & 0.2 & 0.1 & 0.05 \\
\hline \multirow{3}{*}{$W_{l}=0.0$} & 0.8 & 16.00 & 10.67 & 6.86 & 4.00 & 1.78 & 0.84 \\
\hline & 0.4 & 8.00 & 5.33 & 3.43 & 2.00 & 0.89 & 0.42 \\
\hline & 0.2 & 4.00 & 2.67 & 1.71 & 1.00 & 0.44 & 0.21 \\
\hline \multirow{3}{*}{$W_{l}=0.2$} & 1.0 & 30.70 & 19.43 & 12.05 & 6.85 & 2.98 & 1.40 \\
\hline & 0.8 & 24.56 & 15.54 & 9.64 & 5.48 & 2.39 & 1.12 \\
\hline & 0.2 & 6.14 & 3.89 & 2.41 & 1.37 & 0.60 & 0.28 \\
\hline \multirow{5}{*}{$W_{l}=0.4$} & 1.0 & 59.06 & 33.60 & 19.56 & 10.65 & 4.50 & 2.09 \\
\hline & 0.8 & 47.25 & 26.88 & 15.64 & 8.52 & 3.60 & 1.67 \\
\hline & 0.6 & 35.43 & 20.16 & 11.73 & 6.39 & 2.70 & 1.25 \\
\hline & 0.4 & 23.62 & 13.44 & 7.82 & 4.26 & 1.80 & 0.84 \\
\hline & 0.2 & 11.81 & 6.72 & 3.91 & 2.13 & 0.90 & 0.42 \\
\hline
\end{tabular}

focus is set as $f$, the distance between the photographing center and the base level is $H$, and $h$ is the altitude difference. In addition, $f / H, B x / H, h / H$ respectively stand for the ratio between focal length, component $B x$, altitude difference and $H$. The ratio between focal length and $H$ is 0.02 . Horizontal parallax differences with different photographic parameters are shown in Table $\mathbf{3}$.

From Table 3, it is clear to see that the angle $w$ is directly proportional to the horizontal parallax difference. As the angle increases, the horizontal parallax difference increases as well. If the angle of the original image increases, the horizontal parallax difference of corresponding image pair on horizontal image will be amplified. Especially, when the altitude difference displacement is evident the horizontal parallax difference of corresponding image pair should be paid more attention. The above experimental data again testify that the altitude difference displacement is the fundamental cause for horizontal parallax difference. With the decreasing of the ratio $h / H$, the horizontal parallax difference decreases. When the ratio $h / H$ is less than a certain value, the horizontal parallax difference is nearly negligible. Therefore, it proves that algorithm based on separate relative orientation is restricted only by altitude difference displacement, while the other mosaic algorithms are restricted both by altitude difference displacement and by vertical parallax. For this reason, the algorithm proposed in this paper is certainly advantaged.

Suppose $f / H=0.05$ and the photographic azimuth angles are $-0.2,-0.3,0.2$; Then, compare the registration effect of the algorithm proposed in this paper with the algorithm based on perspective projection transformation model [15]. The data collected form the experiment is shown in Table 4.

Table 4 shows that under the same photographic condition, the maximum horizontal parallax generated in perspective projection transformation model is larger than that generated in the model in this paper. In addition, vertical parallax still exists in perspective projection transformation model, but not in ideal image pair model. To verify the experimental result, several other experimental data are set. And the result is in accordance with the above experiment.

\section{CONCLUSION}

The paper intensively studies the transformation model in image registration and proposes an algorithm that applies relative orientation model in photogrammetry to image mosaic. The ideal image pair is generated by using of relative orientation, and the vertical parallax of image pairs to be jointed is eliminated. By match the corresponding image pairs of the generated ideal image pairs on the basis of one 
Table 4. Comparison of horizontal parallax between perspective projection and ideal image pair model.

\begin{tabular}{|c|c|c|c|c|c|c|c|}
\hline \multirow{2}{*}{$\begin{array}{l}\text { Mosaic } \\
\text { Method }\end{array}$} & \multirow{2}{*}{$B x / H$} & \multicolumn{6}{|c|}{$h / H$} \\
\hline & & 0.5 & 0.4 & 0.3 & 0.2 & 0.1 & 0.05 \\
\hline \multirow{5}{*}{$\begin{array}{c}\text { Perspective } \\
\text { Projection } \\
\text { Model }\end{array}$} & 1.0 & 68.42 & 50.37 & 35.45 & 22.22 & 10.43 & 5.24 \\
\hline & 0.8 & 67.83 & 49.86 & 34.67 & 21.59 & 9.81 & 4.89 \\
\hline & 0.6 & 65.69 & 47.71 & 32.83 & 19.68 & 9.67 & 4.69 \\
\hline & 0.4 & 57.58 & 41.61 & 27.56 & 16.57 & 7.65 & 4.94 \\
\hline & 0.2 & 45.57 & 27.82 & 18.85 & 10.54 & 4.92 & 3.68 \\
\hline \multirow{5}{*}{$\begin{array}{l}\text { Ideal } \\
\text { Image } \\
\text { Pair } \\
\text { Model }\end{array}$} & 1.0 & 52.25 & 33.50 & 21.54 & 12.56 & 5.58 & 2.64 \\
\hline & 0.8 & 40.20 & 26.80 & 17.23 & 10.05 & 4.47 & 2.12 \\
\hline & 0.6 & 30.15 & 20.10 & 12.92 & 7.54 & 3.35 & 1.59 \\
\hline & 0.4 & 20.10 & 13.40 & 8.61 & 5.02 & 2.23 & 1.06 \\
\hline & 0.2 & 10.05 & 6.70 & 4.31 & 2.51 & 1.12 & 0.53 \\
\hline
\end{tabular}

dimension, the margins of jointed pictures fit neatly into each other. Experiments show that this algorithm has an advantage over other existing algorithms in registration error and calculated amount. However, algorithm based on relative orientation cannot completely remove the influence of horizontal parallax on image mosaic, and this problem needs further study.

\section{CONFLICT OF INTEREST}

The authors confirm that this article content has no conflict of interest.

\section{ACKNOWLEDGEMENTS}

Declared none.

\section{REFERENCES}

$\mathrm{N}$. Wang, and C. Wang, "A edge-local image mosaic algorithm based on SIFT", Microelectronics \& Computer, vol. 29, no. 1, pp. 35-37, 2012.

[2] T. Jiang, G. Zhu, and A. Sun, "Review of Research Status Of Panoramic Image Mosaic Technology", Journal of Chongqing Technology and Business University (Natural Science Edition), vol. 29, no. 12, pp. 60-71, 2012.

[3] Y. Li, and Z. Hua, "Image mosaics algorithm based on salient region matching," Computer Engineering and Applications, vol. 48, no. 10 , pp. 204-216, 2012

[4] X. Liu, and Y. Zhou, "Patch-based SAR image automatic registration,” Computer Engineering, vol. 33, no. 4, pp. 176-178, 2007.
[5] C.D. Kuglin, and D.C. Hines, "The Phase Correlation Image Alignment Method", In: IEEE International Conference on Cinematie and Society, 1975, pp. 163-165.

[6] S. Zuo, X. Guo, J. Wan, and B Yang, "Wide baseline parallax image mosaic algorithm," Computer Engineering, vol.33, no. 10, pp. 175-177, 2007.

[7] X. Yuan, and Z. Wu, "A method of epipolar image generation based on POS data", Geomatics and Information Science of Wuhan University, vol. 33, no. 6, pp. 560-564, 2008.

[8] Q. Sen, and J. Zhu, "Improved SIFT-based bidirectional image matching algorithm" Mechanical Science and Technology for Aerospace Engineering, vol. 26, no. 9, pp. 1179-1182, 2007.

[9] D.G. Lowe, "Distinctive image features from scale invariant key points" International Journal of Computer Vision, vol. 60, no. 2, pp. 91-110, 2004.

[10] M.J. Smiht, and D.W.G. Park, "Towards a new approach for absolute and exterior orientation," Photogrammetric Record, vol. 16, no. 94 , pp. 617-623, 1999.

[11] Y. Zhang, and B. Hu, "Relative orientation based on multiple conjugate features" Acta Geodaetica et Cartographica Sinica, vol. 40, no.2, pp.194-199, 2011.

[12] $\mathrm{H} . \mathrm{Li}$, and $\mathrm{Y}$. Chen, "Generating method of the stereo epiporlarline images based on geometric correction" Journal of Shandong Institute of Architecture and Engineering, vol.19, no.4, pp. 45-48, 2004.

[13] H. Bay, T. Tuytelaars, and L. V. Gool, "SURF: Speeded up robust features", In: Proceedings of the European Conference on Computer Vision, Springer-Verlag Berlin Heidelberg, 2006, pp.404-407.

[14] M.A. Fischler, and R.C. Bolles, "Random sample consensus: a paradigm for model fitting with applications to image analysis and automated cartography", Communications of the ACM, vol. 24, no. 6, pp. 381-345, 1981.

[15] Z. Tao, Y. Wei, W. Zuo, "Perspective projection model in system calibration process," Ordnance Industry Automation, vol. 30, no. 3, pp. $30-35,2011$

(c) Yi-fei and Yi; Licensee Bentham Open.

This is an open access article licensed under the terms of the Creative Commons Attribution Non-Commercial License (http://creativecommons.org/licenses/by-nc/3.0/) which permits unrestricted, non-commercial use, distribution and reproduction in any medium, provided the work is properly cited. 